Technical Note

\title{
Head Losses in Junction Manholes for Free Surface Flows in Circular Conduits
}

\author{
Michael Pfister ${ }^{1}$ and Corrado Gisonni, F.ASCE ${ }^{2}$
}

\begin{abstract}
Former studies on combining flows resulted in an efficient layout of sewer junctions operated under supercritical approach flow conditions. Straight extensions allowed a reduction in the shock wave heights generated by the merging flows, so that the global discharge capacity was significantly increased. Herein, an extensive experimental campaign is presented on a physical model with the aforementioned layout, although with generalized geometrical conditions now including various conduit diameters. The effects of the main parameters governing the energy losses for combining flows were ascertained to enhance the information available from the literature. The results and their analysis provide a basis for the prediction of energy losses at junction manholes with different upstream and lateral conduit diameters and various flow conditions. DOI: 10.1061/(ASCE)HY.1943-7900.0000895. @ 2014 American Society of Civil Engineers.
\end{abstract}

Author keywords: Head loss; Hydraulic structures; Junction manhole; Sewer hydraulics; Shock waves; Supercritical flows.

\section{Introduction}

Channel junctions represent a crucial hydraulic structure for natural and built open-channel flow systems. The hydraulic features of flow through a junction are governed by a large set of geometrical and hydraulic parameters, so that it is challenging to formulate an analytical approach to evaluate this phenomenon.

For design discharges, open-channel systems must generally operate under free-surface flow through junctions to prevent surge phenomena and abrupt transitions from free-surface to pressurized flow. This is typically the case for combined sewer systems, being fundamental urban infrastructures. The latter must accomplish a twofold task: namely, to prevent outflow through the manhole covers involving (1) dry-weather flows for hygienic reasons, and (2) storm water runoff causing flooding of urban areas and threats for public health and safety. Sewers constitute a collection system including numerous combining junctions, normally localized in particular manholes. Moreover, junctions are frequent in hydraulic structures, irrigation, and drainage systems.

A hydraulic analysis of this structure is particularly challenging if supercritical approaching flows occur, combined with shock waves, whereas the hydraulic features of subcritical combining flow have been thoroughly investigated in the past, with particular reference to rectangular cross sections which are prone to theoretical approaches. In general, the usual assumptions of nearly uniform flow and negligible boundary friction within the control volumes are accepted (Ramamurthy et al. 1988). Recently, the peculiar condition of surcharged junction manholes has been investigated for rectangular closed conduits (Ramamurthy and Zhu 1997),

\footnotetext{
${ }^{1}$ Research and Teaching Associate, Laboratory of Hydraulic Constructions, Ecole Polytechnique Fédérale de Lausanne, CH-1015 Lausanne, Switzerland (corresponding author). E-mail: michael.pfister@epfl.ch

${ }^{2}$ Professor, Dept. of Civil Engineering, Design, Construction and Environment, Second Univ. Naples, 81031 Aversa, Italy. E-mail: corrado .gisonni@unina2.it

Note. This manuscript was submitted on June 26, 2013; approved on February 26, 2014; published online on April 28, 2014. Discussion period open until September 28, 2014; separate discussions must be submitted for individual papers. This technical note is part of the Journal of Hydraulic Engineering, (C) ASCE, ISSN 0733-9429/06014015(6)/\$25.00.
}

for circular pipes (Zhao et al. 2006), and for case studies (Zhao et al. 2004), finally providing important results in terms of flow patterns description and energy losses evaluation. A crucial issue was to establish the maximum free-surface elevation within a surcharged manhole to prevent blown-off manhole covers, sewer geysering, urban flooding, and structural failure.

Extensive experimental data are available to estimate local head losses in pressurized conduit flow, with particular reference to combining flows (Vogel 1926, 1928; Gardel and Rechsteiner 1971; Idel'cik 1986; Oka and Ito 2005). The estimation of local energy losses for free-surface flows is often based, under certain conditions, on a similarity between open-channel flow and pressurized conduit flow. Indeed, pressurized flow can be considered as a special case of free-surface flow if the Froude number is $<0.7$. Then, the loss coefficients obtained for local head loss in pressurized flows are also adopted for the corresponding open-channel flow situation (Hager 2010; Gisonni and Hager 2012).

Uniform flow approaching junction manholes may be supercritical depending on the local topography, particularly for bottom slopes exceeding roughly some per mille units (i.e., 0.004-0.005 under ordinary roughness conditions). For such flow conditions, shock waves occur due to local singularities (e.g., contractions, expansions, bends, or even changes in roughness and bottom slope). Despite the challenging phenomenon (Chow 1959), including three-dimensional (3D) effects and presence of air-water mixture flow, few systematic studies were published.

Supercritical flows at junction manholes were mainly investigated using physical models, with particular attention to the shock wave features and manhole choking for rectangular channels (Schwalt and Hager 1995) and for circular sewers (Del Giudice and Hager 2001; Gisonni and Hager 2002b), proposing a design procedure for such manholes. However, no systematic investigation on head losses in supercritical combining flows is available, so far. This circumstance is probably due to the fact that energy losses are generally regarded as a minor concern for supercritical flows, given their excess of energy. Typically, discharge capacity and shock wave occurrence are considered as primary design issues. Nevertheless, an evaluation of energy losses induced by standard sewer appurtenances is relevant for an adequate modeling of openchannel systems, such as sewer networks (Yen 1986). 


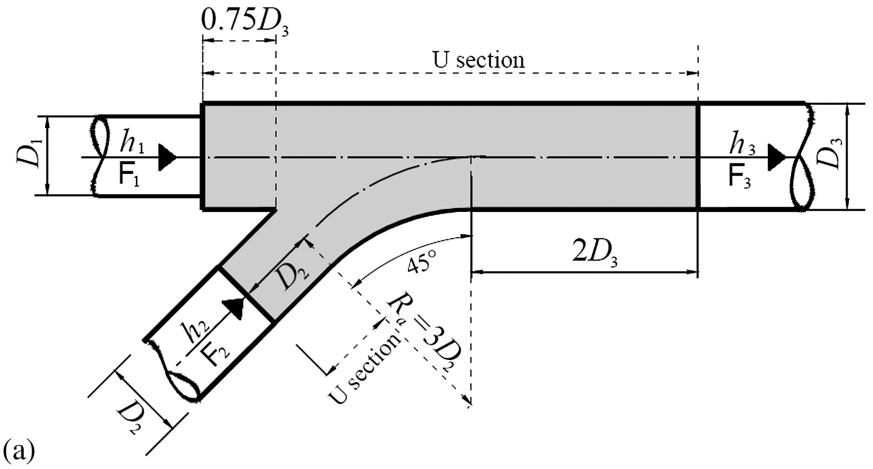

(a)
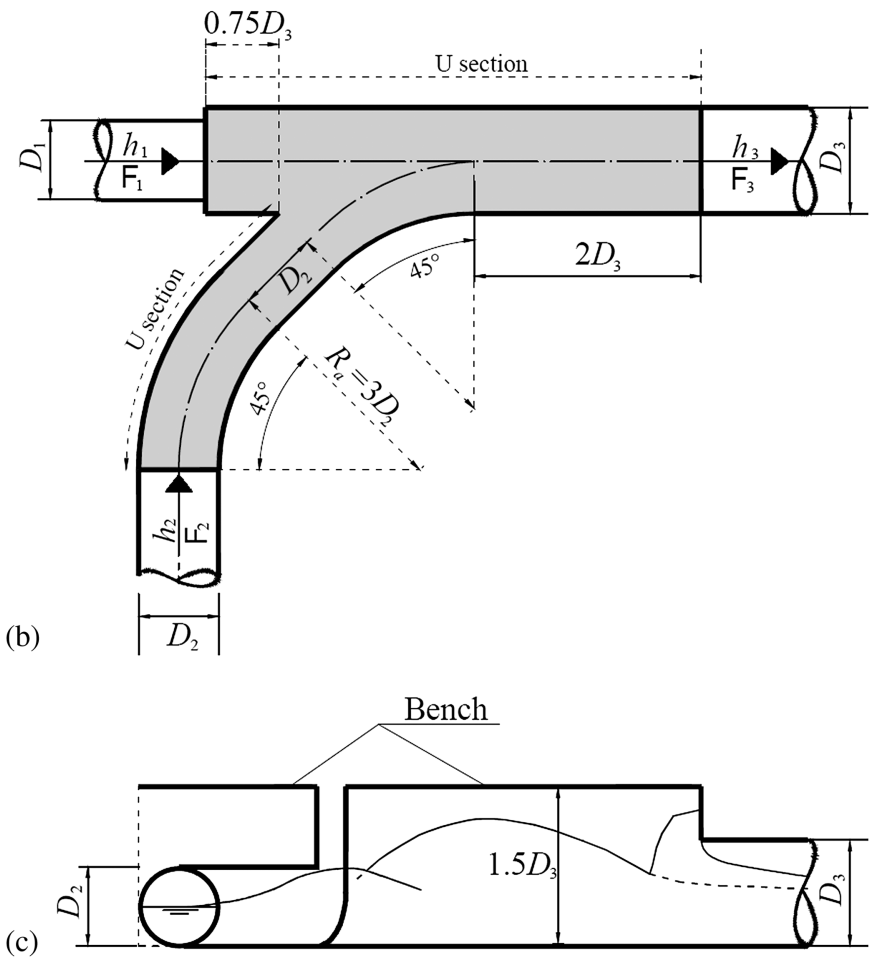

Fig. 1. Scheme of junction manhole: (a) plan view for $\theta=45^{\circ}$; (b) plan view for $\theta=90^{\circ}$; (c) section (gray $=\mathrm{U}$-shaped portion; white $=$ circular conduit portion)

\section{Experiments}

\section{Physical Model}

An experimental campaign was performed at the Laboratory of Hydraulic Constructions ( $\mathrm{LCH}$ ) of Ecole Polytechnique Fédérale de Lausanne (EPFL) (Simos 2012; Gökok 2013; Niedermann 2013). Starting from the junction manhole geometry implemented by Del Giudice and Hager (2001) and Gisonni and Hager (2002b), additional physical models were set up allowing for a general variation of the inflow features and the junction geometry, under junction angles of $45^{\circ}$ and $90^{\circ}$. The main addition consisted of the presence of varying diameters for the combining conduits, all having circular sections (Fig. 1). The outlet pipe diameter was fixed to $D_{3}=0.240 \mathrm{~m}$, and the approach pipe diameters $D_{1}$ and $D_{2}$ were $0.123,0.190$, and $0.240 \mathrm{~m}$ (including all possible combinations; Fig. 2). Here the subscripts 1, 2, and 3 indicate the upstream straight, lateral, and outlet branch of the junction, respectively.

The geometrical features of the models were as follows (Gisonni and Hager 2002a, b; Fig. 1):

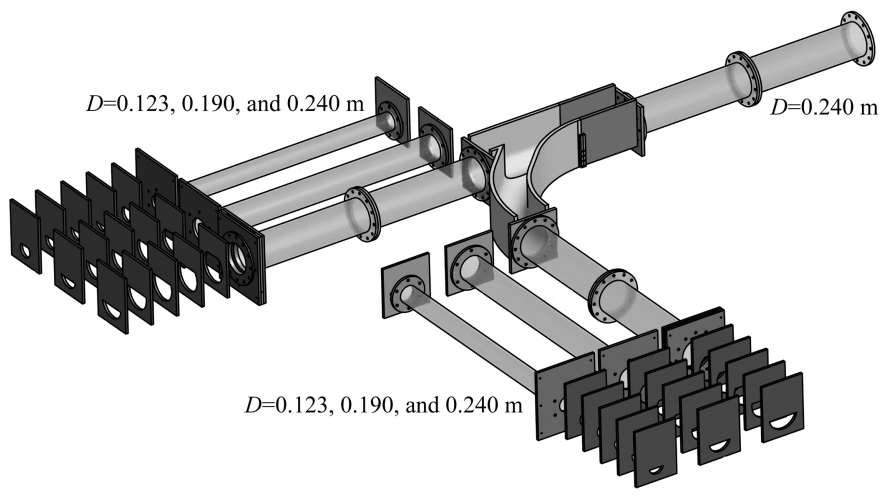

Fig. 2. Overview of model elements including jet boxes, conduits, and junction (note that the geometry of the lateral branch is shown only for $\left.D_{2}=0.24 \mathrm{~m}\right)$

- The capacity of the junction manhole is improved by including a so-called manhole extension, consisting of a straight U-shaped portion of length $2 \cdot D_{3}$ upstream of the manhole outlet;

- The U-shaped section is configured with $1.5 \cdot D_{3}$ high benches, across the junction; and

- The lateral conduit has an axial curvature radius of $R_{a}=3 \cdot D_{2}$, with a straight portion of length $1 \cdot D_{2}$, upstream of the junction point, ending with a junction angle of $45^{\circ}$.

The bottom slope of the physical model was negligible and can be assumed to compensate wall friction. Approaching flows were generated through jet boxes consisting of a sandwich-type structure, also including flow straighteners, allowing for smooth transition from the pressurized supplying circuit to free-surface flow with a fixed flow depth $h$. It was thus possible to impose independent variations of the approach velocity $V$ and the filling ratio $y$ in both combining pipes.

In total, more than 600 tests were recorded, aiming to characterize the main flow features of manhole junctions and, particularly, the head losses. The flow process is basically governed by the following parameters: filling ratios $y_{i}=h_{i} / D_{i}$, Froude numbers $\mathrm{F}_{i}$, and diameter ratios $\beta_{i}=D_{i} / D_{3}$ of the two approach branches, i.e., $i=1,2$. It has to be remarked that, for a given discharge $Q$, the Froude number can be approximated as $\mathrm{F}=Q /\left(g D h^{4}\right)^{1 / 2}$, with an accuracy of some $\pm 3 \%$ for $0.20 \leq y \leq 0.90$ (Hager 2010; Gisonni and Hager 2012).

Experimental runs covered a wide range of the main parameters, such as $0.10 \leq y_{i} \leq 1.0$ and $0.2 \leq \mathrm{F}_{i} \leq 15.7$ (Table 1). Discharges $(Q)$ between 3 and $100 \mathrm{l} / \mathrm{s}$ were supplied per each inflow conduit and measured to $\pm 0.5 \%$ full scale (FS) accuracy with inductive discharge measurement devices (IDM) installed in both supply conduits. Flow depths were measured using point gauges with a reading accuracy of $\pm 0.5 \mathrm{~mm}$ and a typical depth reading of $\pm 1 \mathrm{~mm}$ due to free-surface fluctuations of supercritical flows.

The extended experimental campaign allowed for a detailed description of the main hydraulic features, including free-surface profiles and discharge capacity. Hereafter, the focus is on junction head losses for both subcritical and supercritical flow conditions.

\section{Basic Features of Junction Flow}

Junction manholes may present various flow conditions (Hager 2010), among which the following are the most important in practice: 
Table 1. Experimental Range of Main Parameters

\begin{tabular}{|c|c|c|c|c|c|c|}
\hline Junction angle & $\beta_{1}$ & $y_{1}$ & $\mathrm{~F}_{1}$ & $\beta_{2}$ & $y_{2}$ & $\mathrm{~F}_{2}$ \\
\hline $45^{\circ}$ & $0.51,0.79,1.00$ & $0.17-0.92$ & $0.2^{\mathrm{a}}-10.6$ & $0.51,1.00$ & $0.10-0.94$ & $0.2^{\mathrm{a}}-10.3$ \\
\hline $90^{\circ}$ & $0.51,0.79,1.00$ & $0.19-1.00$ & $0.2^{\mathrm{a}}-15.7$ & $0.51,0.79,1.00$ & $0.10-1.00$ & $0.2^{\mathrm{a}}-6.5$ \\
\hline
\end{tabular}

${ }^{a}$ The flow conditions with zero discharge in one of the two upstream conduits $\left(\mathrm{F}_{i}=0\right.$ for $i=1$ or 2$)$ are not included in Table 1.

- Supercritical approach flow in both branches;

- Subcritical flow in lateral, and supercritical flow in straight branch;

- Supercritical flow in lateral, and subcritical flow in straight branch; and

- Subcritical flow in both branches.

All mentioned conditions were tested herein. The two extreme cases are considered, with either sub- or supercritical flow occurring in both approach branches. The flow patterns are substantially different for these two conditions, as described hereafter.

Fig. 3 shows the typical flow pattern for subcritical flow in both branches. It can be noted that the surface is smooth, and the lateral inflow does not perturb the upstream straight flow. The formation of a separation zone immediately downstream of the junction point is visible, with development of macroscopic vortices that constitute the core of the energy dissipation mechanism. A similar flow structure can be observed in both $45^{\circ}$ and $90^{\circ}$ junction manholes.

Fig. 4 shows typical features for supercritical combining flows at $45^{\circ}$ and $90^{\circ}$ junction manholes. Three types of shock waves are distinguished (Gisonni and Hager 2002b):

1. On the wall opposite of the lateral branch due to flow impingement; generally, this is the highest wave developing within a junction manhole;
2. Along the curved portion of the lateral branch, which has similar features to that formed within a bend manhole; the straight portion with length $1 \cdot D_{2}$ inhibits further growth of the wave downstream of the $45^{\circ}$ curved portion; and

3. The swell generated by the flow impacting the outlet section of the manhole; if the swell height is large, the combined flow may choke, thus causing abrupt transition from free-surface flow to pressurized flow.

The only evident difference between the $45^{\circ}$ and the $90^{\circ}$ junction is the absence of the bend shock wave for the $45^{\circ}$ junction that developed along the curved portion of the $90^{\circ}$ lateral branch.

\section{Scale Effects}

Physical modeling is mandatory to study complex flow phenomena, such as supercritical combining flows. However, the model behavior may differ from prototype because of scale effects. Based on the Froude similitude, a preliminary investigation concluded that $h \geq 0.04-0.05 \mathrm{~m}$ allows for scale effects to be considered negligible (Gökok 2013). Then the Reynolds numbers $R=$ $4 R_{h} V / \nu$ of all considered tests becomes $2.7 \cdot 10^{4} \leq \mathrm{R}_{1} \leq 1.1 \cdot 10^{6}$ and $3.0 \cdot 10^{4} \leq \mathrm{R}_{2} \leq 4.9 \cdot 10^{5}$, with $R_{h}=$ hydraulic radius and $\nu=$ water viscosity. These limitations are in agreement with those

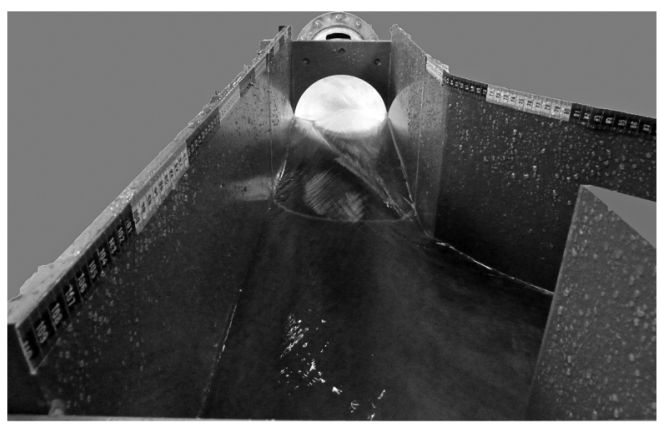

(a)

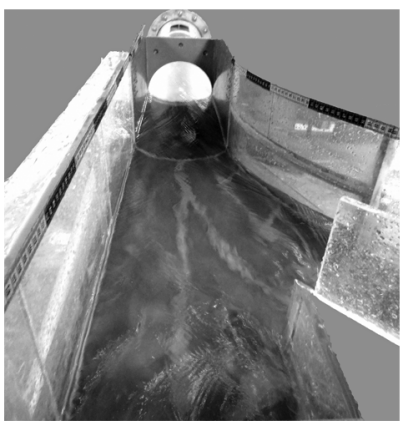

(b)

Fig. 3. Upstream views of subcritical flow patterns in (a) $90^{\circ}$ junction manhole $\left(y_{1}=0.68, F_{1}=0.26, \beta_{1}=1, y_{2}=0.85\right.$, $F_{2}=0.31, \beta_{2}=0.79$ ); (b) $45^{\circ}$ junction manhole $\left(y_{1}=0.88, \mathrm{~F}_{1}=0.28, \beta_{1}=0.79, y_{2}=0.72, \mathrm{~F}_{2}=0.24, \beta_{2}=1\right.$ ) (images by authors)

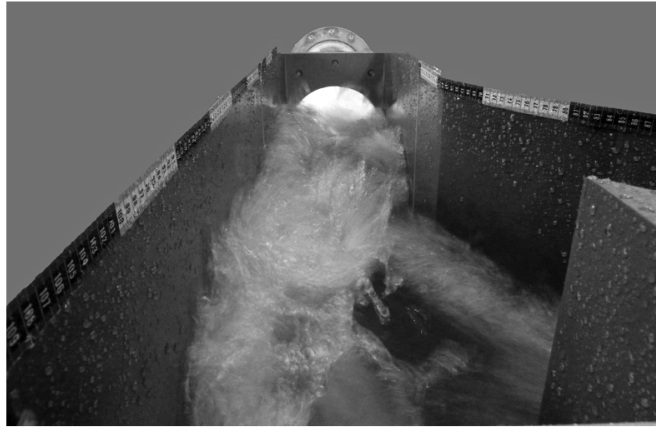

(a)

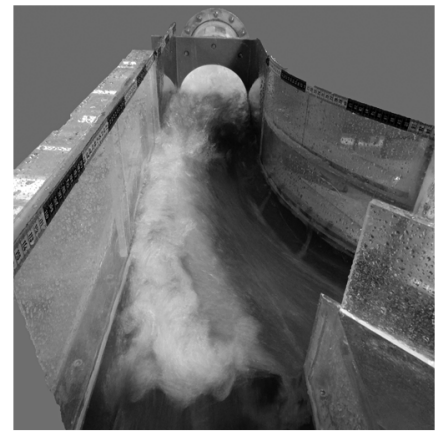

(b)

Fig. 4. Upstream views of supercritical flow patterns in (a) $90^{\circ}$ junction manhole $\left(y_{1}=0.30, \mathrm{~F}_{1}=4.1, \beta_{1}=1, y_{2}=0.50, \mathrm{~F}_{2}=2.6, \beta_{2}=0.79\right.$ ); (b) $45^{\circ}$ junction manhole $\left(y_{1}=0.31, \mathrm{~F}_{1}=6.2, \beta_{1}=0.79, y_{2}=0.39, \mathrm{~F}_{2}=2.0, \beta_{2}=1\right)$ (images by authors) 
proposed by Del Giudice and Hager (2001), Gisonni and Hager (2002b), and Pfister and Chanson (2012). Even if this limitation was contemplated for the experimental runs overall, few exceptions were accepted, with negligible deviations as compared to the rest of the data.

\section{Energy Losses: Theoretical Background}

The flow process for combining or dividing flows is governed by the cardinal principles of conservations of mass, energy, and momentum. Based on these equations, it is possible to develop analytical expressions for the local energy losses and corresponding coefficients.

The local energy losses $\Delta H$ induced by the junction may be expressed through the energy equation, written in terms of energy fluxes

$$
Q_{1} H_{1}+Q_{2} H_{2}-Q_{3} H_{3}=Q_{3} \Delta H
$$

where the total head $H_{i}$ at the reference section $i$ (Fig. 1) is given as

$$
H_{i}=z_{i}+h_{i}+\frac{V_{i}^{2}}{2 g}
$$

with invert elevation $z_{i}$, flow depth $h_{i}$, and average velocity $V_{i}$.

In the following, as generally assumed, the differences in bottom elevations are considered negligible (i.e., $z_{i}=0 \mathrm{~m}$ ), as are the friction losses (only local losses are considered accordingly). The head loss coefficients $\xi$ are conventionally defined as

$$
\begin{gathered}
\xi_{1,3}=\frac{H_{1}-H_{3}}{V_{3}^{2} / 2 g} \\
\xi_{2,3}=\frac{H_{2}-H_{3}}{V_{3}^{2} / 2 g} \\
\xi_{j}=\frac{\Delta H}{V_{3}^{2} / 2 g}
\end{gathered}
$$

where the subscript $j$ refers to the global manhole energy loss. Based on Eq. (1), the global head loss coefficient $\xi_{j}$ can be expressed as

$$
\xi_{\mathrm{j}}=\frac{Q_{1}}{Q_{3}} \xi_{1,3}+\frac{Q_{2}}{Q_{3}} \xi_{2,3}
$$

In addition to Eq. (1), the momentum conservation equation gives

$$
\rho\left(Q_{1} V_{1}+Q_{2} V_{2} \cos \theta-Q_{3} V_{3}\right)=\sum P_{x}
$$

where $\theta=$ the junction angle and $P_{x}$ is the components of the pressure forces along the main flow direction.

The exchanges of momentum result in energy transfer from the main stream (larger discharge and velocity) to the merging stream. It is thus possible that the merging flow may leave the junction manhole with energy content larger than it had upstream. This circumstance implies the possibility of having negative loss coefficient, because the main stream undergoes energy losses while transferring part of its momentum to the merging flow.

It is possible to deduce generalized equations for combining flows by linking the energy, momentum, and continuity equations. In particular, the components of the pressure forces $P_{x}$ in Eq. (5) may assume different and complicate formulations, depending on manhole geometry and flow conditions. However, it has to be remarked that it is not evident how to evaluate pressure forces for supercritical flows across a bend or junction manhole, due to the presence of shock waves and significant curvature effects (i.e., nonhydrostatic pressure distribution). This is the case of the curved walls (Fig. 1) guiding the lateral flow within the benches of the junction.

For combining flows, the following generalized expressions for the head loss coefficients result:

$$
\begin{aligned}
\xi_{1,3}= & c_{1,3}+a_{1,3}\left[1-2 \frac{A_{3}}{A_{1}}\left(\frac{Q_{1}}{Q_{3}}\right)^{2}-2 \frac{A_{3}}{A_{2}}\left(\frac{Q_{2}}{Q_{3}}\right)^{2} \cos \theta\right. \\
& \left.+\left(\frac{A_{3}}{A_{1}}\right)^{2}\left(\frac{Q_{1}}{Q_{3}}\right)^{2}\right] \\
\xi_{2,3}= & c_{2,3}+a_{2,3}\left[1-2 \frac{A_{3}}{A_{1}}\left(\frac{Q_{1}}{Q_{3}}\right)^{2}-2 \frac{A_{3}}{A_{2}}\left(\frac{Q_{2}}{Q_{3}}\right)^{2} \cos \theta\right. \\
& \left.+\left(\frac{A_{3}}{A_{2}}\right)^{2}\left(\frac{Q_{2}}{Q_{3}}\right)^{2}\right]
\end{aligned}
$$

with the coefficients $a_{1,3}, c_{1,3}, a_{2,3}$, and $c_{2,3}$ to be determined experimentally, given that the components of the pressure forces $P_{x}$ cannot be expressed analytically due to complex features of manhole geometry and approaching flows.

For the particular case of surcharged manholes, with both the inlet pipes and the outlet pipe pressurized, assuming that the piezometric heads of the approach flows are equal to the water level in the manhole (i.e., hydrostatic pressure distribution), Zhao et al. (2006) supposed a simple definition for the terms $P_{x}$ in Eq. (5) and derived the analytical expressions of the loss coefficients $\xi^{*}$

$$
\begin{aligned}
& \xi_{1,3}^{*}=1-2 \frac{A_{3}}{A_{1}}\left(\frac{Q_{1}}{Q_{3}}\right)^{2}-2 \frac{A_{3}}{A_{2}}\left(\frac{Q_{2}}{Q_{3}}\right)^{2} \cos \theta+\left(\frac{A_{3}}{A_{1}}\right)^{2}\left(\frac{Q_{1}}{Q_{3}}\right)^{2} \\
& \xi_{2,3}^{*}=1-2 \frac{A_{3}}{A_{1}}\left(\frac{Q_{1}}{Q_{3}}\right)^{2}-2 \frac{A_{3}}{A_{2}}\left(\frac{Q_{2}}{Q_{3}}\right)^{2} \cos \theta+\left(\frac{A_{3}}{A_{2}}\right)^{2}\left(\frac{Q_{2}}{Q_{3}}\right)^{2}
\end{aligned}
$$

The latter are equivalent to Eqs. $(6 a)$ and $(6 b)$ for the particular case $a_{1,3}=a_{2,3}=1$ and $c_{1,3}=c_{2,3}=0$.

\section{Experimental Results}

The cross-sectional area $A$ and the Froude number $\mathrm{F}$ of circular channels may be approximated as (Hager 2010; Gisonni and Hager 2012)

$$
\begin{aligned}
& A=D^{2}\left(\frac{h}{D}\right)^{1.5}=D^{2} y^{1.5} \\
& \mathrm{~F}=\frac{Q}{\sqrt{g D h^{4}}}=\frac{Q}{\sqrt{g D^{5} y^{4}}}
\end{aligned}
$$

allowing for an adaption of Eqs. (6a) and (6b) to

$$
\xi_{1,3}=c_{1,3}+a_{1,3}\left[1-2 \frac{\mathrm{F}_{1}^{2} y_{1}^{2.5} \beta_{1}^{3}+\mathrm{F}_{2}^{2} y_{2}^{2.5} \beta_{2}^{3} \cos \theta}{\mathrm{F}_{3}^{2} y_{3}^{2.5}}+\beta_{1} \frac{\mathrm{F}_{1}^{2} y_{1}}{\mathrm{~F}_{3}^{2} y_{3}}\right]
$$




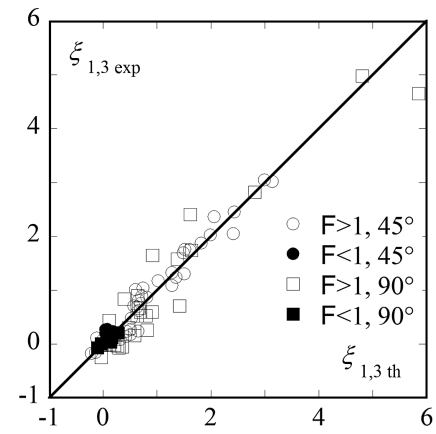

(a)

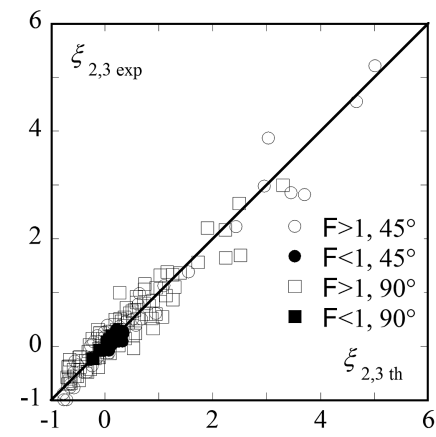

(b)
Fig. 5. Comparison of experimental and theoretical $\xi$ values for the (a) main; (b) lateral branch

$$
\xi_{2,3}=c_{2,3}+a_{2,3}\left[1-2 \frac{\mathrm{F}_{1}^{2} y_{1}^{2.5} \beta_{1}^{3}+\mathrm{F}_{2}^{2} y_{2}^{2.5} \beta_{2}^{3} \cos \theta}{\mathrm{F}_{3}^{2} y_{3}^{2.5}}+\beta_{2} \frac{\mathrm{F}_{2}^{2} y_{2}}{\mathrm{~F}_{3}^{2} y_{3}}\right]
$$

In Fig. 5, the head loss coefficients $\xi_{1,3 \exp }$ and $\xi_{2,3 \exp }$ (for suband supercritical flows) as derived from model measurements (subscript exp) are plotted against the theoretical (subscript th) $\xi_{1,3 \text { th }}$ and $\xi_{2,3 \text { th }}$ values resulting from Eqs. (10a) and (10b). The values of the coefficients $a_{1,3}, c_{1,3}, a_{2,3}$, and $c_{2,3}$ are listed in Table 2 for both sub- and supercritical approach flow conditions. Note that the head loss coefficients for subcritical flow conditions were never larger than $\xi_{1,3}=0.25$ and $\xi_{2,3}=0.35$ for the $45^{\circ}$ junction, and $\xi_{1,3}=0.30$ and $\xi_{2,3}=0.25$ for the $90^{\circ}$ junction. The coefficients of determination were larger than 0.90 , despite the macroturbulent nature of combining supercritical flows.

An effective inflow angle $\delta$ had to be accounted for within the momentum Eq. (5), as discussed by Ramamurthy and Zhu (1997) and Hager (1987). According to the junction geometry (Fig. 1), the lateral flow is influenced by the straight extension of length $1 \cdot D_{2}$. For the $90^{\circ}$ junction manhole, the data analysis confirmed that a lateral inflow angle of $\delta=45^{\circ}$ had to be assumed in Eqs. (10a) and $(10 \mathrm{~b})$, instead of the geometrical junction angle $\theta=90^{\circ}$.

The coefficients in Table 2 allow for some remarks:

- For any flow condition, the absolute values of the coefficients for the $45^{\circ}$ junction manhole are systematically larger than for the $90^{\circ}$ junction manhole. This circumstance is an effect of the manhole geometries which affect the term $P_{x}$ in Eq. (5).

- For the $90^{\circ}$ junction manhole, with supercritical flow conditions, it is possible to assume $a_{1,3}=a_{2,3}=0.70$ and $c_{1,3}=c_{2,3}=$ 0.15 , without introducing significant error in the evaluation of $\xi_{1,3}$ and $\xi_{2,3}$.

- For both $45^{\circ}$ and $90^{\circ}$ junction manholes, with subcritical flow condition, $c_{1,3}$ and $c_{2,3}$ are negative, but with almost the same absolute values for the supercritical flow condition.

Table 2. Empirical Coefficients of Eqs. (10a) and (10b)

\begin{tabular}{lrrrr}
\hline Junction angle & $a_{1,3}$ & $c_{1,3}$ & $a_{2,3}$ & $c_{2,3}$ \\
\hline $45^{\circ}$ & & & & \\
Supercritical flow & 0.72 & 0.27 & 0.83 & 0.16 \\
Subcritical flow & 0.91 & -0.30 & 0.75 & -0.16 \\
$90^{\circ}$ & & & & \\
Supercritical flow & 0.70 & 0.15 & 0.68 & 0.16 \\
Subcritical flow & 0.80 & -0.13 & 0.54 & -0.08 \\
\hline
\end{tabular}

For subcritical flow in the junction, the values of $y_{3}$ and $H_{3}$ follow from the backwater curve of the downstream conduit. These serve to derive the flow parameters $y_{1}$ and $H_{1}$, and $y_{2}$ and $H_{2}$, respectively. To reduce the number of iteration steps when solving Eq. (10), one may assume $\xi_{1,3}=0.25$ and $\xi_{2,3}=0.35$ as a first approximation. During the physical model tests, these values were never exceeded for subcritical conditions. For supercritical flow conditions in the junction, the inflow conditions are defined by the uniform flow regime of the two upstream conduits (or their drawdown curves), whereas the values $y_{3}$ and $H_{3}$ are a priori unknown. Again, the iteration process linked to Eq. (10) may be simplified by estimating $y_{3}$ with Eq. (11). The latter follows from the physical model tests, with $P_{3}=\left(Q_{1}+Q_{2}\right) /\left(g D_{3}^{5}\right)^{0.5}$ as the conduit Froude number:

$$
y_{3}=\frac{2 \mathrm{P}_{3}}{\mathrm{~F}_{1} y_{1} \beta_{1}+\mathrm{F}_{2} y_{2} \beta_{2}}
$$

\section{Conclusions}

A comprehensive experimental campaign was conducted to investigate the local head losses of combining flows at $45^{\circ}$ and $90^{\circ}$ junction manholes on circular conduits, with various diameters and in the presence of sub- and supercritical approaching flows. The analysis of the experimental results, along with the application of the basic principles of mass, energy, and momentum conservation, provided theoretical expressions for the head loss coefficients which fit well the data measured on the physical models.

In principle, approximate and simpler expressions could be derived from Eqs. (10a) and (10b) to facilitate practical calculation. In this case, the disadvantages of renouncing their physical background and accepting marginal errors for the head losses evaluation should be taken into account. The expressions of the head loss coefficients provide an important tool for the numerical modeling of open-channel flows in sewer systems and drainage networks, according to the classical computation procedures outlined for supercritical and subcritical flow by Yen (1986) and Hager (2010).

\section{Acknowledgments}

The measurements were conducted by Mr. Dimitri Simos, Mr. Timur Gökok, and Mr. Emmanuel Niedermann as Master Theses at LCH. The project was financially supported by the City of Zurich (ERZ Entsorgung + Recycling Zurich). The Laboratory of Hydraulics, Hydrology and Glaciology (VAW) of ETH Zurich provided model elements.

\section{Notation}

The following symbols are used in this paper:

$A=$ cross-sectional area;

$D=$ conduit diameter;

$\mathrm{F}=$ Froude number;

$g=$ gravitational acceleration;

$H=$ total head;

$h=$ flow depth;

$\mathrm{P}=$ conduit Froude number;

$P=$ pressure force;

$\mathrm{R}=$ Reynolds number;

$R_{h}=$ hydraulic radius;

$Q=$ discharge;

$V=$ average cross-sectional velocity; 
$y=h / D$ filling ratio;

$\beta_{\iota}=D_{i} / D_{3}$ diameter ratio, for $i=1,2$;

$\gamma=$ unit weight of water;

$\Delta H=$ head loss;

$\delta=$ effective lateral inflow angle;

$\theta=$ junction angle;

$\nu=$ water viscosity;

$\xi=$ head loss coefficient;

$\xi^{*}=$ head loss coefficient for surcharged manhole; and

$\rho=$ water density.

\section{Subscripts}

$\exp =$ experimentally derived;

$j=$ junction manhole;

th $=$ following from theory;

$1=$ straight branch;

2 = lateral branch; and

$3=$ downstream branch.

\section{References}

Chow, V. T. (1959). Open channel hydraulics, McGraw-Hill, New York.

Del Giudice, G., and Hager, W. H. (2001). "Supercritical flow in $45^{\circ}$ junction manhole.” J. Irrig. Drain. Eng., 10.1061/(ASCE)0733-9437(2001) 127:2(100), 100-108.

Gardel, A., and Rechsteiner, G. F. (1971). "Les pertes de charge dans les branchements en Té des conduites de section circulaire." Communication du Laboratoire d'hydraulique, Ecole Polytechnique Fédérale de Lausanne (EPFL), 23, 1-29 (in French).

Gisonni, C., and Hager, W. H. (2002a). "Supercritical flow in manholes with a bend extension." Exp. Fluids, 32(3), 357-365.

Gisonni, C., and Hager, W. H. (2002b). "Supercritical flow in the $90^{\circ}$ junction manhole." Urban Water, 4(4), 363-372.

Gisonni, C., and Hager, W. H. (2012). Idraulica dei sistemi fognari: Dalla teoria alla pratica, Collana: UNITEXT - Ingegneria, Springer, Milan, Italy (in Italian).

Gökok, T. (2013). "Physical model investigation of supercritical flow in junction manholes." M.Sc. thesis, Laboratory of Hydraulic Constructions (LCH), Ecole Polytechnique Fédérale de Lausanne (EPFL), Lausanne, Switzerland.
Hager, W. H. (1987). "Discussion of 'Separation zone at open-channel junctions' by J. L. Best and I. Reid." J. Hydraul. Eng., 10.1061/ (ASCE)0733-9429(1987)113:4(539), 539-543.

Hager, W. H. (2010). Wastewater hydraulics: Theory and practice, Springer, Berlin.

Idel'cik, I. E. (1986). Handbook of hydraulic resistance, Hemisphere Publishing Corporation, Washington, DC.

Niedermann, E. (2013). "Physical model investigation of supercritical flow in $45^{\circ}$ junction manholes." M.Sc. thesis, Laboratory of Hydraulic Constructions (LCH), Ecole Polytechnique Fédérale de Lausanne (EPFL), Lausanne, Switzerland.

Oka, K., and Ito, H. (2005). "Energy losses at tees with large area ratios." J. Fluids Eng., 127(1), 110-116.

Pfister, M., and Chanson, H. (2012). "Discussion of scale effects in physical hydraulic engineering models." J. Hydraul. Res., 50(2), 244-246.

Ramamurthy, A. S., Carballada, L. B., and Tran, D. M. (1988). "Combining open channel flow at right angled junctions." J. Hydraul. Eng., 10.1061/ (ASCE)0733-9429(1988)114:12(1449), 1449-1460.

Ramamurthy, A. S., and Zhu, W. (1997). "Combining flows in $90^{\circ}$ junctions of rectangular closed conduits." J. Hydraul. Eng., 10.1061/ (ASCE)0733-9429(1997)123:11(1012), 1012-1019.

Schwalt, M., and Hager, W. H. (1995). "Experiments to supercritical junction flow." Exp. Fluids, 18(6), 429-437.

Simos, D. (2012). "Model investigation of supercritical flow in junction manholes with variable conduit diameters." M.Sc. thesis, Laboratory of Hydraulic Constructions ( $\mathrm{LCH}$ ), Ecole Polytechnique Fédérale de Lausanne (EPFL), Lausanne, Switzerland.

Vogel, G. (1926). "Untersuchungen über den Verlust in rechtwinkligen Rohrverzweigungen." Mitteilungen des Hydraulischen Instituts der Technischen Hochschule München, 1, 75-90 (in German).

Vogel, G. (1928). "Untersuchungen über den Verlust in rechtwinkeligen Rohrverzweigungen." Mitteilungen des Hydraulischen Instituts der Technischen Hochschule München, 2, 61-64 (in German).

Yen, B. C. (1986). "Hydraulics of sewers." Advances in Hydroscience, Vol. 14, Academic, New York, 1-122.

Zhao, C. H., Zhu, D. Z., and Rajaratnam, N. (2004). "Supercritical sewer flows at a combining junction: A case study of Edworthy trunk junction, Calgary, Alberta." J. Environ. Eng. Sci., 3(5), 343-353.

Zhao, C. H., Zhu, D. Z., and Rajaratnam, N. (2006). "Experimental study of surcharged flow at combining sewer junctions." J. Hydraul. Eng., 10 .1061/(ASCE)0733-9429(2006)132:12(1259), 1259-1271. 